\title{
Lipid saturation and head group composition have a pronounced influence on the membrane insertion equilibrium of amphipathic helical polypeptides
}

\author{
Evgeniy Salnikov, ${ }^{1}$ Christopher Aisenbrey ${ }^{1}$ and Burkhard Bechinger ${ }^{1,2, *}$ \\ ${ }^{1}$ University of Strasbourg / CNRS, UMR7177 Chemistry Institute, Membrane Biophysics \\ and NMR, Strasbourg, France \\ ${ }^{2}$ Institut Universitaire de France
}

*Corresponding author: Burkhard Bechinger, 4 Blaise Pascal, F-67000, Strasbourg, France. Tel.: +33 3688513 03; E-mail: bechinge@unistra.fr

Key words: helix topology, lipid order parameter, lipophobic effect, hydrophobic mismatch, molecular shape, magainin, PGLa, solid-state NMR, supported lipid bilayer

Running title: lipid unsaturation drives helix insertion 


\begin{abstract}
The histidine-rich peptides of the LAH4 family were designed using cationic antimicrobial peptides such as magainin and PGLa as templates. The LAH4 amphipathic helical sequences exhibit a multitude of interesting biological properties such as antimicrobial activity, cell penetration of a large variety of cargo and lentiviral transduction enhancement. The parent peptide associates with lipid bilayers where it changes from an orientation along the membrane interface into a transmembrane configuration in a $\mathrm{pH}$-dependent manner. Here we show that LAH4 adopts a transmembrane configuration in fully saturated DMPC membranes already at $\mathrm{pH} 3.5$, i.e. much below the $\mathrm{pK}_{\mathrm{a}}$ of the histidines whereas the transition $\mathrm{pH}$ in POPC correlates closely with histidine neutralization. In contrast in POPG membranes the inplanar configuration is stabilized by about one $\mathrm{pH}$ unit. The differences in $\mathrm{pH}$ can be converted into energetic contributions for the in-plane to transmembrane transition equilibrium, where the shift in the transition $\mathrm{pH}$ due to lipid saturation corresponds to energies which are otherwise obtained by the exchange of several cationic with hydrophobic residues. A similar dependence on lipid saturation has also been observed when the PGLa and magainin antimicrobial peptides interact within lipid bilayers suggesting that the quantitative evaluation presented in this paper also applies to other membrane polypeptides.
\end{abstract}

\title{
Highlights
}

*Lipid saturation strongly drives the interface-to-transmembrane realignment

*The energetic contribution of lipid saturation corresponds to removing numerous charges from the amino acid sequence

*Anionic lipids stabilize the interfacial localization of cationic amphipathic peptides.

\section{TOC graphics}

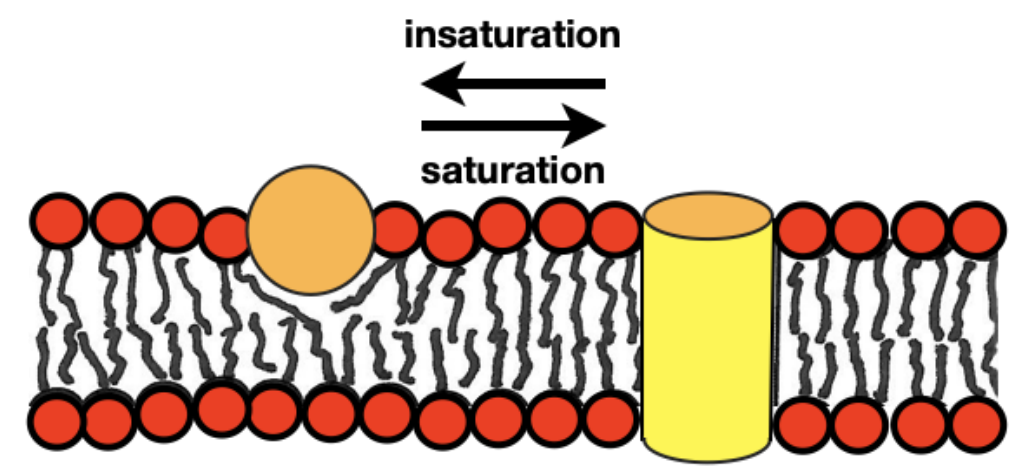




\section{Abbreviations used}

ATR FTIR attenuated total reflection Fourier transform infra red

$\mathrm{CP} \quad$ cross polarization

DMPC 1,2-dimyristoyl-sn-glycero-3-phosphocholine

DMPE 1,2-dimyristoyl-sn-glycero-3-phosphoethanolamine

IP in-plane

MALDI-TOF matrix-assisted laser desorption ionization - time of flight

NMR nuclear magnetic resonance

PC 1,2-diacyl-sn-glycero-3-phosphocholine

PE 1,2-diacyl-sn-glycero-3-phosphoethanolamine

POPC 1-palmitoyl-2-oleoyl-sn-glycero-3-phosphocholine

POPE 1-palmitoyl-2-oleoyl-sn-glycero-3-phosphoethanolamine

POPG 1-palmitoyl-2-oleoyl-sn-glycero-3-phospho-(1'-rac-glycerol) sodium salt

RP-HPLC reversed-phase high performance liquid chromatography

TFA trifluoroacetic acid

TFE trifluoroethanol

TM transmembrane 


\section{Introduction}

Helical polypeptide sequences constitute important building blocks of membrane proteins [1$3]$ and membrane-associated peptides are also active as independent units [4, 5]. When polypeptide sequences interact with lipid bilayers they can adopt transmembrane or surfaceassociated orientations, with important consequences for their function [6]. These topologies exchange with each other through reversible chemical equilibria and topological variations that depend on the environmental conditions have been observed [7-10]. The topologies of membrane polypeptide domains have been tested qualitatively using accessibility measurements [11] or in a quantitative manner by biophysical methods such as ATR FTIR and solid-state NMR spectroscopies [12-14]. Biophysical experiments allow one to also determine intermolecular interactions, supramolecular arrangements and oligomerization of polypeptides within liquid crystalline bilayers. For example, biophysical studies have resulted in important insight into the functioning of peptides [15-18] and on the aggregation behavior of transmembrane or membrane-proximal sequences [19-21].

As expected, very hydrophobic sequences have been found to preferentially adopt transmembrane alignments whereas amphipathic sequences tend to interact with the interface of lipid bilayers [6, 22-25]. However, depending on the detailed conditions, alamethicin, a peptide devoid of any charges has also been found at surface orientations [26], and cationic amphipathic sequences have been observed in transmembrane configurations [8, 27].

This data are indicative that the membrane topologies of these sequences are not determined by the amino acid composition alone [28]. In contrast, there are many more interaction contributions that govern the equilibria of polypeptides where the location and topology changes from buffer $\Leftrightarrow$ membrane interface (in-plane) $\Leftrightarrow$ membrane inserted (transmembrane). These transitions are accompanied by structural transitions including the formation of folded structures when random coil sequences in aqueous solution adopt helical structures in the presence of lipid bilayers [29, 30]. Related topological transitions are also observed for beta-sheet structures [31, 32]. Furthermore, oligomerization and supramolecular organisation can occur which extends the simplified scheme mentioned above $[10,33]$. The interactions that govern topological equilibria of helical sequences have been tested including Leu-Ala model sequences with lysines introduced at strategic positions, polyalanines, hydrophobic sequences of different lengths or membranes of different hydrophobic thickness [34-36].

When the membrane alignment of the antimicrobial peptide PGLa (Table 1) is investigated the peptide always aligns parallel to the surface of unsaturated membranes $[8,27$, 
37]. However, in fully saturated lipid bilayers the detailed tilt angle not only depends on the peptide-to-lipid ratio, the hydrophobic thickness and the bilayer hydration but also shows a pronounced dependence on the lipid composition [8,38]. A wide range of angles is observed for PGLa which even adopts transmembrane orientations in the presence of magainin 2 (Table 1), another antimicrobial peptide produced by the same frog [8, 27, 37]. When studied in saturated membranes the magainin 2 sequence also shows a somewhat different tilt due to the presence of PGLa although the effects are much less pronounced than for PGLa in the same lipid/peptide mixture [39]. Notably, in an environment of saturated membranes the realignment of a protein domain in the presence of another could have regulatory functions [8] thus a better understanding of such processes is of potential biomedical importance.

\section{TABLE 1: Sequences of peptides discussed in this paper}

$\begin{array}{lllll}\text { LAH4 } & \text { KKALL ALALH HLAHL ALHLA LALKK A-NH } \\ \text { magainin 2 } & \text { GIGKF LHSAK KFGKA FVGEI MNS- } \mathrm{NH}_{2} \\ \text { PGLa } & \text { GMASK AGAIA GKIAK VALKA L- } \mathrm{NH}_{2}\end{array}$

In the past much focus had been given to the composition of the lipid head group in order to explain how these peptides selectively interact with bacterial cell membranes [40]. Later on, the saturation of the lipid fatty acyl chains has been found to make a pronounced difference when the PGLa interactions with membranes are studied [8, 27, 37]. The LAH4 sequence was initially designed (Table 1 ) to better understand and test the orientationdependence and activity of antimicrobial peptides [41]. Thereafter, the LAH4 peptides were also shown to exhibit potent antimicrobial and cell penetrating activities for nucleic acids, proteins, peptides, vaccines, nanodots, adeno associated viruses and more [42]. Furthermore, a derivative of the sequence is explored in clinical settings for its lentiviral transduction enhancement activities [43]. The LAH4 peptides are made up of a leucine-alanine hydrophobic core which is interrupted by four histidines. In an $\alpha$-helical conformation these line up along one face of the helical surface thus an amphipathic structure is formed (Figure S1). At both termini two lysines each increase solubility and serve as interfacial anchoring residues. In solution the histidines are characterized by a $\mathrm{pK}$ a around 6 , therefore the hydrophobic moment and the membrane alignment of the LAH4 peptides is strongly $\mathrm{pH}$ dependent $[12,41]$.

Oriented ${ }^{15} \mathrm{~N}$ solid-state NMR and ATR FTIR spectroscopies of uniaxially oriented POPC lipid bilayers have demonstrated a reversible topological transition of LAH4 from an 
in-planar $(\mathrm{pH}<5.5)$ to a transmembrane state $(\mathrm{pH}>7)$ with a midpoint of $6.1 \pm 0.2[12,41]$. Single amino acid substitutions have been shown to change transition $\mathrm{pH}$ and thereby have allowed the evaluation of the energetic contribution of individual amino acids when they move from the membrane interface to its hydrophobic interior $[44,45]$. When more than one labelled site is explored more accurate tilt angle information has been obtained also for LAH4 at $\mathrm{pH} 5.3[46]$.

In this paper, we investigated in quantitative detail how the transition of LAH4 is affected by the lipid composition, in particular it's fatty acyl chain saturation. Therefore, the $\mathrm{pH}$-dependent topological transition of this helical peptide was investigated in different membrane environments. In particular, the role of the lipid saturation and head group composition was analyzed by investigating in quantitative detail the transmembrane or inplanar alignment of LAH4 using ${ }^{15} \mathrm{~N}$ solid-state NMR spectroscopy [47]. In parallel information on the membrane macroscopic phase properties and the packing of the lipid fatty acyl chains is obtained from ${ }^{31} \mathrm{P}$ and ${ }^{2} \mathrm{H}$ solid-state NMR spectra, respectively [48].

\section{Materials and Methods}

1-palmitoyl-2-oleoyl-sn-glycero-3-phosphocholine (POPC) in its natural abundance or with the palmitoyl chain deuterated were purchased from Coger (Paris, France), 1,2-dimyristoylsn-glycero-3-phosphocholine (DMPC) and 1-palmitoyl-2-oleoyl-sn-glycero-3phosphoglycerol (POPG) and ${ }^{2} \mathrm{H}$-depleted water $(<1 \mathrm{ppm})$ were from Sigma-Aldrich / Merck KGaA (St Quentin Fallavier, France). F-moc protected amino acids were from NovaBiochem / Merck KGaA (Darmstadt, Germany), isotope labeled amino acids from Cortecnet (Voisins les Bretonneux, France), Euriso-top (Paris, France) or Isotec ${ }^{\circledR}$ Sigma-Aldrich/Merck KGaA (St Quentin Fallavier, France), the TentaGel-S-RAM resin used during peptide synthesis was from Rapp Polymer GmbH (Tübingen, Germany).

The peptide sequence of LAH4 is KKALL $\underline{\text { ALALH HLAHL }}$ ALHLA LALKK A$\mathrm{CONH}_{2}$ where the underlined positions Ala6 and Ala16 were labelled with ${ }^{15} \mathrm{~N}$ one at a time. The sequence was prepared by solid-phase peptide synthesis using a Millipore 9050 automatic peptide synthesizer and a four-fold excess of Fmoc protected amino acid as described previously [49].

The peptides were purified by reversed phase HPLC (Gilson, Villiers-le-Bel, France)

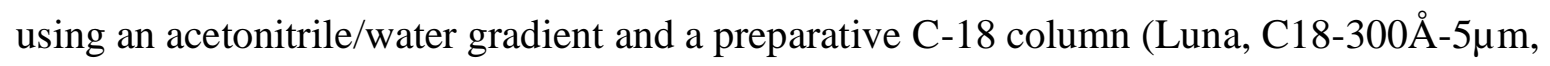
Phenomenex, Le Pecq, France). The identity and purity of the product (>90\%) were analyzed 
by HPLC and MALDI mass spectrometry (MALDI-TOF Autoflex, Bruker Daltonics, Bremen, Germany). Before usage the peptides were lyophilized from a solution in $4 \%$ acetic acid to exchange the trifluoroacetic acid counter ions [50].

\section{Sample Preparation for ${ }^{2} \mathrm{H}$-solid-state NMR measurements}

Non-oriented samples were prepared by mixing POPC/POPC-d $\mathrm{d}_{31}(4.3 \mathrm{mg} / 1.5 \mathrm{mg}, 3 / 1)$ or DMPC/DMPC-d54 $(4.3 \mathrm{mg} / 1.5 \mathrm{mg}, 3 / 1)$ in trifluoroethanol (TFE). TFE was evaporated with a stream of nitrogen and exposure to high vacuum overnight such that a film forms along the walls of the glass tube ( $6 \mathrm{~mm}$ outer diameter). LAH4 $(0.38 \mathrm{mg})$ in $140 \mu \mathrm{lmilliQ}$ water, or for comparison the same amount of water without peptide, was added to each vial, and the $\mathrm{pH}$ was adjusted to the desired value by addition of $50 \mathrm{mM}$ solutions of either $\mathrm{NaOH}$ or $\mathrm{HCl}$. Thereafter, the samples were lyophilized and the lipids suspended in $25 \mu 1$ of the appropriate buffer by vortexing and bath sonication, followed by three freeze/thaw cycles at $-20{ }^{\circ} \mathrm{C}$ and 40 ${ }^{\circ} \mathrm{C}$, respectively, to produce multilamellar vesicles. The buffers were $100 \mathrm{mM}$ Tris (pH 7.5), $100 \mathrm{mM}$ acetate (pH 5.1), or $100 \mathrm{mM}$ citric acid at either $\mathrm{pH} 5.0$ or $\mathrm{pH}$ 3.0. For solid-state NMR measurements the samples were inserted into the solenoidal coil of the solid-state NMR probe. The spectra were recorded at $298 \mathrm{~K}$ for POPC and $310 \mathrm{~K}$ for DMPC samples.

\section{Preparation of supported lipid bilayers for solid-state NMR spectroscopy}

Typically, $3.7 \mathrm{mg}$ of LAH4 labelled with ${ }^{15} \mathrm{~N}$ at either the alanine 6 or alanine 16 position was dissolved in $300 \mu \mathrm{L}$ milliQ water. The $\mathrm{pH}$ was adjusted by addition of a few microliters of 1 $\mathrm{M}$ solutions of $\mathrm{NaOH}$ or $\mathrm{HCl}$. Thereafter the peptide solution was added to $55 \mathrm{mg}$ lipid, vortexed, bath-sonicated for 5 minutes, exposed to three cycles between $40^{\circ} \mathrm{C}$ and $-20^{\circ} \mathrm{C}$. Thereafter, the $\mathrm{pH}$ was controlled and readjusted if necessary. The suspension was loaded onto 23 ultrathin glass plates $(8 \times 18$ mm, thickness 00; Marienfeld, Lauda-Königshofen, Germany), left overnight under the hood to dry and equilibrated at $93 \%$ relative humidity at $37{ }^{\circ} \mathrm{C}$ for two days. The $\mathrm{pH}$ value of the lipid/peptide samples was again tested from a small part of the sample that was mixed with $300 \mu 1$ milliQ water. After equilibration, the glass plates were stacked on top of each other, wrapped with Teflon tape and sealed in plastic wrapping [50].

\section{Solid-state NMR spectroscopy}

Proton-decoupled ${ }^{31} \mathrm{P}$ solid-state NMR spectra at $121.576 \mathrm{MHz}$ were recorded using a Bruker Avance wide-bore 300 solid-state NMR spectrometer equipped with a commercial double- 
resonance flat-coil probe (Bruker, Rheinstetten, Germany). A Hahn-echo pulse sequence [51] was used were the $\pi / 2$ pulse was $7 \mu$ s, the spectral width $100 \mathrm{kHz}$, the echo delays $100 \mu \mathrm{s}$, the acquisition time $10.2 \mathrm{~ms}$, and the recycle delay $3 \mathrm{~s} .85 \% \mathrm{H}_{3} \mathrm{PO}_{4}$ at 0 ppm was used as an external reference. The temperature was set to $25^{\circ} \mathrm{C}$ for POPC, and to $37{ }^{\circ} \mathrm{C}$ for DMPC and POPG samples. A Lorentz apodization of $50 \mathrm{~Hz}$ was applied before the Fourier transform.

${ }^{2} \mathrm{H}$ solid-state NMR spectra of deuterated POPC- $\mathrm{d}_{31}$ or DMPC-d $\mathrm{d}_{54}$ were recorded by applying a quadrupolar pulse-echo sequence [52]. The recycle delay was $0.3 \mathrm{~s}$, the echo time $100 \mu \mathrm{s}$, the dwell time $0.5 \mu \mathrm{s}$ and the $\pi / 2$ pulse $5 \mu \mathrm{s}$. An exponential apodization with line broadening of $300 \mathrm{~Hz}$ was applied before Fourier transformation of the free induction decay. The temperature was set to $25^{\circ} \mathrm{C}$ for POPC and $37^{\circ} \mathrm{C}$ for DMPC samples.

Proton-decoupled ${ }^{15} \mathrm{~N}$ cross-polarization (CP) spectra were recorded either at 76.016 $\mathrm{MHz}$ or at $30.432 \mathrm{MHz}$ on Bruker Avance wide bore NMR spectrometers using a cross polarization pulse sequence and an e-free (at the17.6 Tesla magnet [53]) or a standard (at 7 Tesla) flat-coil double-resonance probe [54]. The spectral width, acquisition time, CP contact time, and recycle delay time were $100 \mathrm{kHz}, 3.9 \mathrm{~ms}, 0.4 \mathrm{~ms}$, and $2 \mathrm{~s}$, respectively. The $\mathrm{B}_{1}$ field strength was $35 \mathrm{kHz}$ during $\mathrm{CP}$, for the ${ }^{1} \mathrm{H} \pi / 2$ pulse and the SPINAL-64 heteronuclear decoupling [55]. A Lorentzian apodization of $200 \mathrm{~Hz}$ was applied before the Fourier transform. An external reference of ${ }^{15} \mathrm{NH}_{4} \mathrm{Cl}$ was used for calibration of the ${ }^{15} \mathrm{~N}$ chemical shift scale (39.3 ppm; [56]). The temperature was set to $25^{\circ} \mathrm{C}$ for POPC and $37{ }^{\circ} \mathrm{C}$ for DMPC samples.

\section{Quantitative evaluation of the LAH4 topological transition}

The transmembrane fraction was obtained from oriented ${ }^{15} \mathrm{~N}$ solid-state NMR spectra by comparing the integrals corresponding to the transmembrane region (140 to $240 \mathrm{ppm}$ ) with the total signal intensity (40 to $240 \mathrm{ppm}$ ). The corresponding $\mathrm{pH}$ titration curves were analyzed according to [45]:

$$
\left.P_{T M}=\frac{[T M]}{[T M]+\left[I P^{o}\right]+\left[I P^{c h}\right]}=\frac{1}{1+e^{\frac{\Delta G}{R T}}\left(1+e^{2.3\left(p K_{a}-p H\right)}\right)^{4}} \text { (equation } 1\right) .
$$

The $\mathrm{pK}_{\mathrm{a}}$ value was fixed to 6.1 except for POPG where an apparent $\mathrm{pK}_{\mathrm{a}}$ of 7.1 was taken into consideration.

\section{Deuterium order parameters}

The deuterium order parameters $(\mathrm{S} \mathrm{CD})$ of the $\mathrm{CD}_{2}$ and $\mathrm{CD}_{3}$ groups was calculated by dePakeing [57] according to: $\mathrm{S}_{\mathrm{CD}}^{\mathrm{i}}=\frac{4}{3} \frac{\mathrm{h}}{\mathrm{e}^{2} \mathrm{qQ}} \Delta^{\mathrm{i}} \mathrm{v}$, where $\Delta^{\mathrm{i}} \mathrm{v}$ is the quadrupolar splitting of segment $\mathrm{i}$ 
and $\left(\mathrm{e}^{2} \mathrm{qQ} / \mathrm{h}\right)$ is the static quadrupole coupling constant $(167 \mathrm{kHz})$ for $\mathrm{C}-\mathrm{D}$ deuterons [58]. When establishing order parameter profiles, based on early studies of pure lipid bilayers labelled with ${ }^{2} \mathrm{H}_{2}-\mathrm{C}$ at individual segments one at a time, it is assumed that the order parameters diminishes in a continuous fashion from the membrane interface to the membrane interior.

Statistical considerations: The quality of the spectra allows one to determine the quadrupolar splitting with about $1 \mathrm{kHz}$ accuracy. This corresponds to an error of 0.01 for the order parameters and 0.014 for the relative order parameters. Here we compare order parameter profiles encompassing up to $n=13$ data points rather than individual order parameters. In this case Student's $t$ can be calculated from the sample average $(\bar{D})$ and the standard deviation $\left(S_{d}\right)$ of the pair-wise differences between corresponding points of two data

sets according to $t=\frac{\bar{D}}{s_{d}} \sqrt{n}$, where $n$ is the number of data points/differences [59]. From reference t-values it can be estimated that relative order parameters profiles that are at least 0.02 units apart are different with high probability.

\section{Results}

Here we investigated the effect of lipid fatty acyl chain and head group composition on the topological transition of the LAH4 peptide from in-planar to transmembrane alignments. In order to monitor peptide orientation relative to the membrane normal the LAH4 sequence was prepared with a single ${ }^{15} \mathrm{~N}$ label at either the Ala- 6 or the Ala-16 position. The peptide was reconstituted into supported lipid bilayers and studied by proton-decoupled ${ }^{15} \mathrm{~N}$ solid-state NMR spectroscopy. When the sample is inserted with its normal parallel to the magnetic field direction of the NMR spectrometer ${ }^{15} \mathrm{~N}$ chemical shifts $<100 \mathrm{ppm}$ occur at helix alignments approximately parallel to the membrane surface (in-plane, IP state) whereas ${ }^{15} \mathrm{~N}$ chemical shifts around $200 \mathrm{ppm}$ are associated with transmembrane orientations (TM state). Because the chemical shift scale follows a continuous trend intermediate chemical shifts are observed at other tilt angles [47].

The ${ }^{15} \mathrm{~N}$ solid-state NMR spectra of 2 mole\% LAH4 in DMPC exhibit a predominant peak in the range of transmembrane alignments also at acidic $\mathrm{pH}$ with chemical shifts of 218 $\pm 11 \mathrm{ppm}$ at pH 5.3 and $210 \pm 10 \mathrm{ppm}$ and pH 4.9 (Figure 1A,B), where the width of the spectral intensity at half-height is also indicated. When the $\mathrm{pH}$ is further lowered the spectrum at $\mathrm{pH} 3.8$ reveals a close to equal distribution of two intensities at $202 \pm 20 \mathrm{ppm}$ and $77 \pm 19$ 
ppm (Figure 1C). At pH 3.0 a dominant intensity in the in-planar range (79 \pm 16 ppm) (Figure 1D). The ${ }^{31} \mathrm{P}$ solid-state NMR spectra of the same samples exhibit predominant peaks around $30 \mathrm{ppm}$ with intensities extending to $-15 \mathrm{ppm}$ indicative of phospholipid bilayers that are well aligned along the glass plates [48] despite the highly acidic $\mathrm{pH}$ and the orientational dispersion of the peptide (Figure 2A,B).
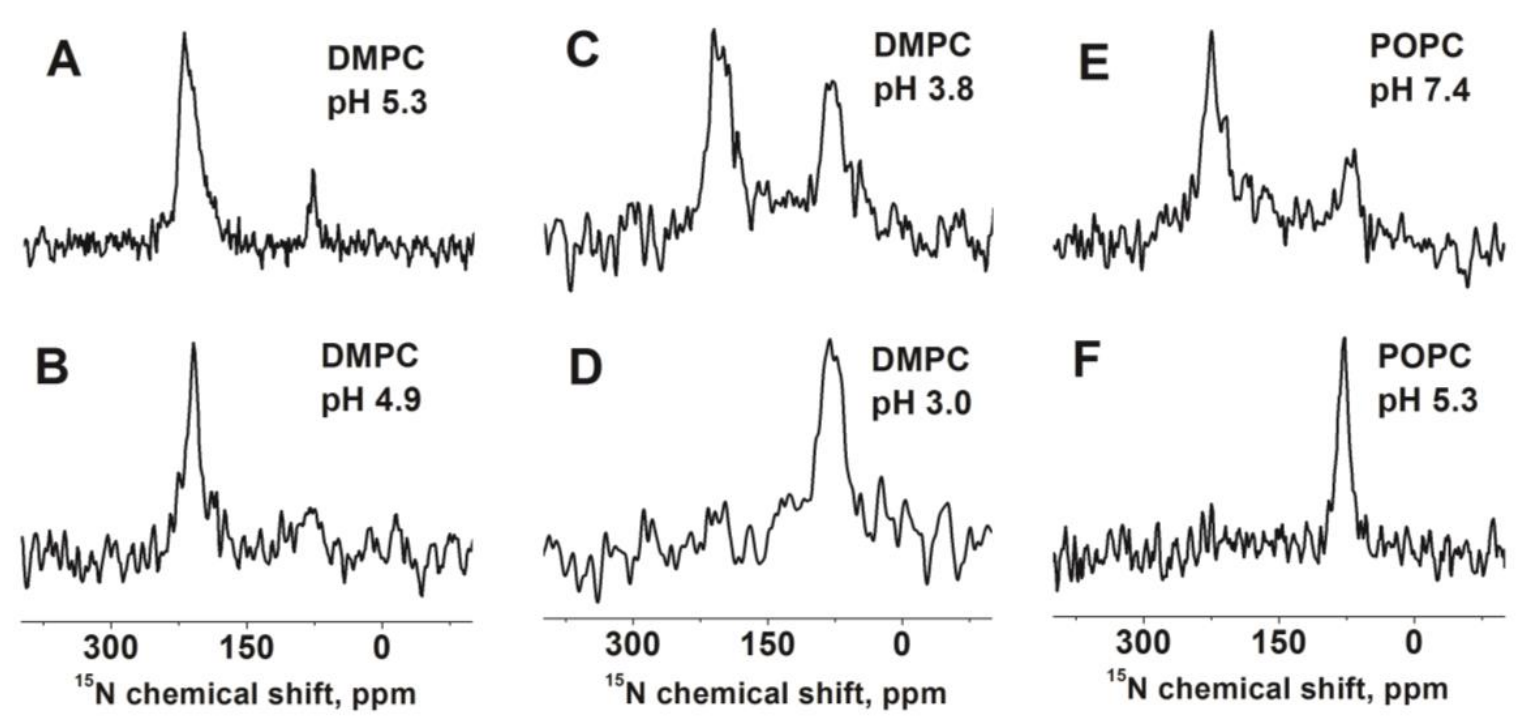

Figure 1: ${ }^{15} \mathrm{~N}$ proton-decoupled spectra of 2 mole $\%\left[{ }^{15} \mathrm{~N}\right.$-Ala16]-LAH4 in mechanically supported DMPC (A-D) or POPC (E, F) bilayers. The pH was adjusted to 5.3 (A), 4.9 (B), 3.8 (C), $3.0(\mathrm{D}), 7.4(\mathrm{E})$, and $5.3(\mathrm{~F})$. The sample normal is parallel to the magnetic field direction of the NMR spectrometer. The spectrum in panel $\mathrm{E}$ is from [60].

Because a large amount of data have already been obtained for LAH4 in POPC at different $\mathrm{pH}[12,41,60]$., here we only investigated a limited number of samples as control (Figure 1E,F). Indeed, the peptide adopts transmembrane alignments at pH $7.4(225 \pm 15$ ppm; Figure 1E) and in-planar orientations at pH 5.3 (79 \pm 8 ppm for Ala 16, Figure 1F and $73.5 \pm 2.5 \mathrm{ppm}$ for Ala 6 [46]) in agreement with the previously measured transition $\mathrm{pH}$ of 6.1 $\pm 0.2[12,41]$. 


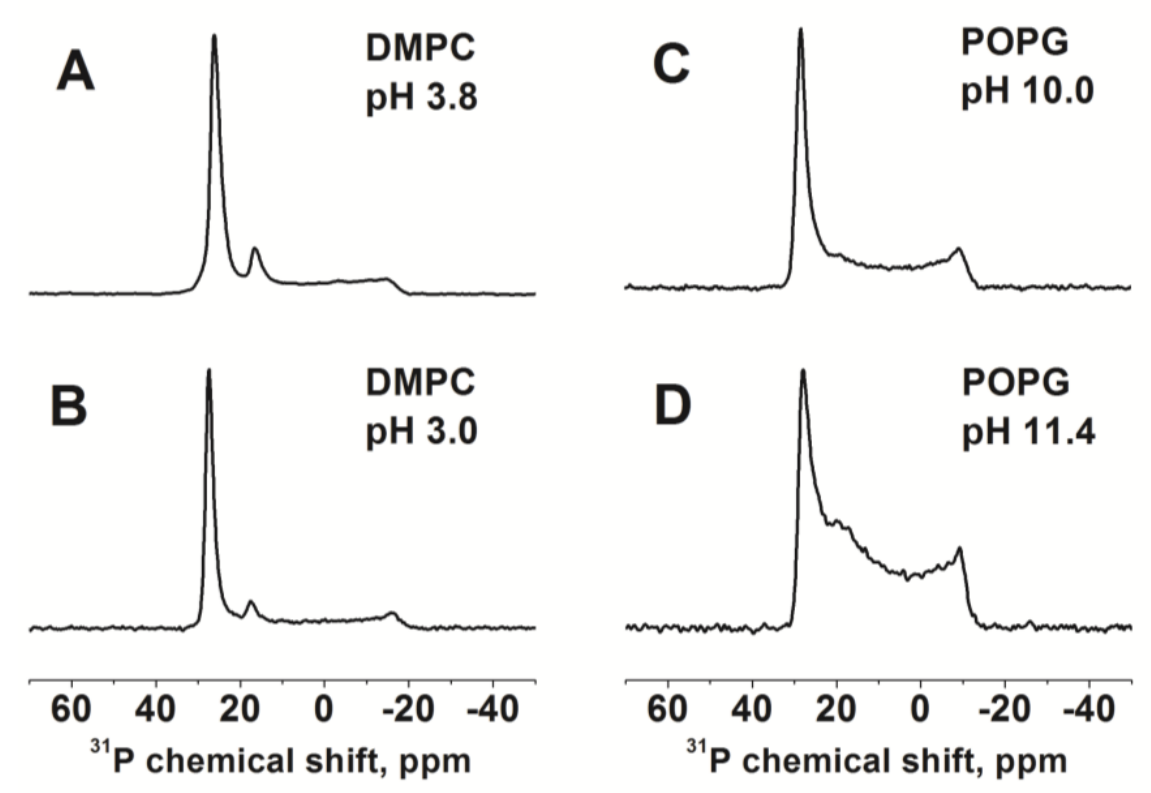

Figure $2 .{ }^{31} \mathrm{P}$ proton-decoupled spectra of 2 mole\% LAH4 in oriented DMPC (A and B) or POPG (C and D) bilayers. The pH was adjusted to 3.8 (A), 3.0 (B), 10.0 (C), and 11.4 (D). The spectra were recorded with the sample normal parallel to the magnetic field direction from the same samples also shown in Figures 1C,D and 4C,D.

This data is summarized in Figure 3 where the transmembrane fraction is shown as a function of $\mathrm{pH}$. The midpoint of the topological transition is $\mathrm{pH}_{50}=3.5$ for LAH4 in DMPC and 6.1 in POPC oriented lipid bilayers.

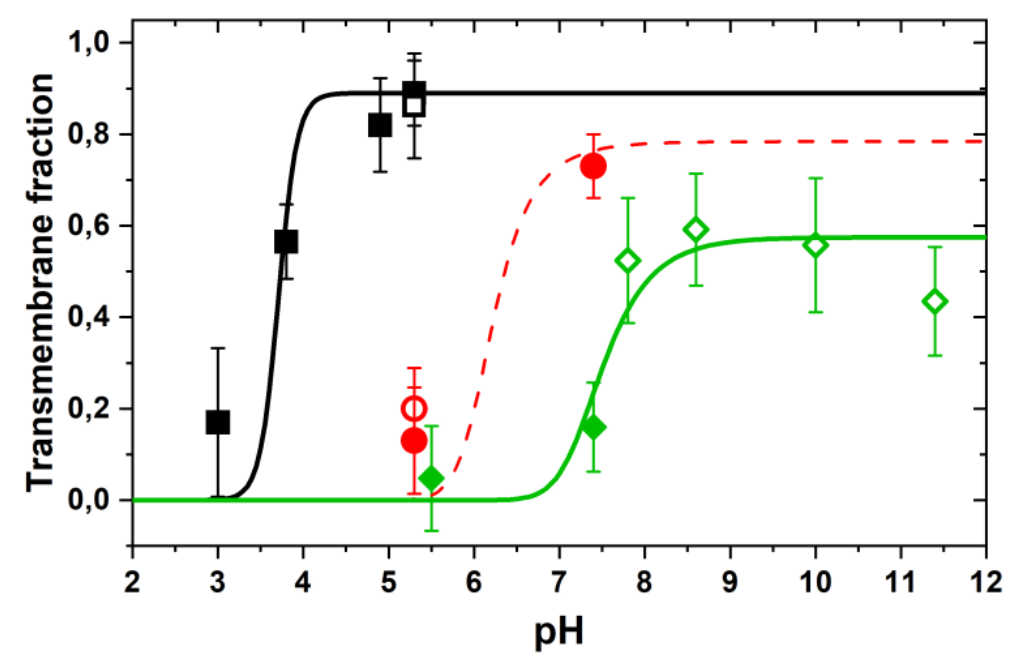

Figure 3: The experimentally measured contribution of the ${ }^{15} \mathrm{~N}$ signal that corresponds to a transmembrane orientation of LAH4 as a function of sample $\mathrm{pH}$ in DMPC (black squares), POPC (red circles) and POPG (green diamonds). Open symbols indicate the results obtained with the ${ }^{15} \mathrm{~N}$ label at position of Ala6, closed ones with ${ }^{15} \mathrm{~N}$-Ala16. The continuous lines show simulations with equation 1 (cf. Methods section) scaled by 0.87 to take into account the 
maximum for the observed transmembrane fraction in DMPC. The $\mathrm{pH}$ was measured from a suspension of $240 \mathrm{mM}$ lipid and the corresponding amount of peptide.

In a next step the LAH4 transition from in-plane to transmembrane was investigated in uniaxially oriented liquid crystalline POPG lipid bilayers. Also, in this case the ${ }^{31} \mathrm{P}$ solid-state NMR spectra indicate that the membranes are reasonably well aligned (Figure 2C,D).

Previously, ${ }^{15} \mathrm{~N}$ chemical shifts of 2 mole $\%{ }^{15} \mathrm{~N}-\mathrm{Ala} 16-\mathrm{LAH} 4$ in POPG of $72.5 \pm 3$ ppm were observed when investigated at $\mathrm{pH} 5.5$ or $\mathrm{pH} 7.4$ indicative that the in-planar alignment is more stable when compared to POPC [60]. Indeed, significant proportions of transmembrane signal intensities were only observed at very basic $\mathrm{pH}$ (Figures 3 and 4). The broad intensities $<100 \mathrm{ppm}$ are indicative of either peptides which do not strongly interact with the oriented membranes and/or contributions of peptides adopting a large range of tilt angles (Figures 4BD). This fraction has a different appearance when compared to the peptide spectrum at $\mathrm{pH}$ 7.8 , where a narrow ${ }^{15} \mathrm{~N}$ peak at $72.5 \mathrm{ppm}$ is indicative of a population aligned along the bilayer surface in a rather homogenous manner (Fig. 4A).
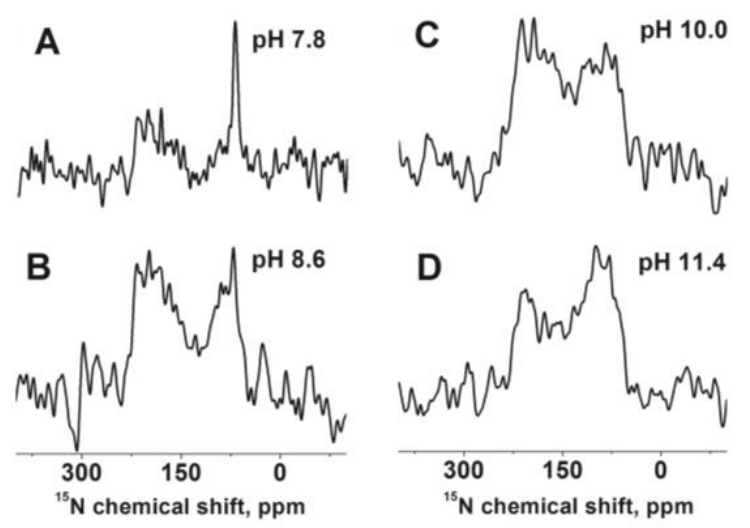

Figure 4: ${ }^{15} \mathrm{~N}$ proton-decoupled spectra of 2 mole $\%\left[{ }^{15} \mathrm{~N}\right.$-Ala16]-LAH4 in oriented POPG bilayers. The $\mathrm{pH}$ was adjusted to 7.8 (A), 8.6 (B), 10.0 (C), and 11.4 (D) at $5.5 \mu 1$ water per $\mathrm{mg}$ of lipid (i.e. at a concentration of $240 \mathrm{mM}$ ).

Finally, the effect of the peptides on the lipid fatty acyl chain order parameters was investigated. These provide valuable information about the packing of the lipids and the effect LAH4 exerts at its different $\mathrm{pH}$-dependent topologies. The order parameters are obtained from ${ }^{2} \mathrm{H}$ solid-state NMR spectra of phospholipids labelled uniformly with ${ }^{2} \mathrm{H}$ at one or both fatty acyl chains (Fig. 5A,D). In this study either a single palmitoyl chain of POPC or both myristoyl chains of DMPC were deuterated. To analyze the effect of LAH4 on the lipid chain mobility and conformation the quadrupolar spectra that compose the ${ }^{2} \mathrm{H}-\mathrm{NMR}$ spectra were 
converted into an order parameter profile (Fig. 5B,E). In order to visualize the segmentdependent changes upon peptide addition the ratio of the order parameter in the presence and in the absence of LAH4 was calculated. These relative order parameters are shown in Figure $5 \mathrm{C}$ and $\mathrm{F}$.
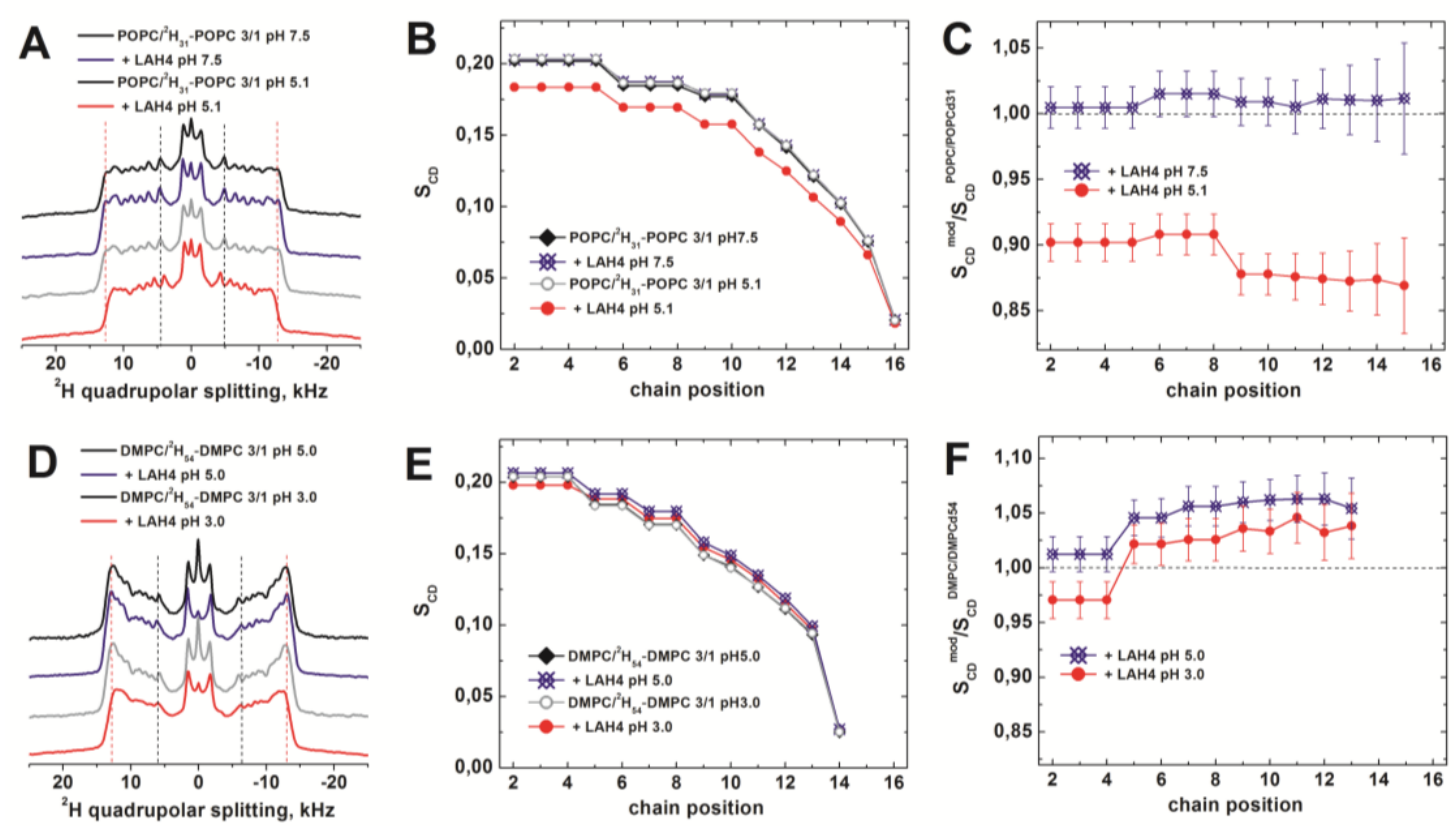

Figure 5. ${ }^{2} \mathrm{H}$ solid-state NMR spectra (A, D) and order parameter profiles (B, C, E, and F) for POPC/ $/ 2 \mathrm{H}_{31}$-POPC 3:1 (A-C) and DMPC/ ${ }^{2} \mathrm{H}_{54}$-DMPC 3:1 (D-F) in the absence and presence of 2 mole\% LAH4 at pH 7.4 or pH 5.1 (A-C), and at pH 5.0 or pH 3.0 (D-F). The temperature was set to $25^{\circ} \mathrm{C}(298 \mathrm{~K})$ for POPC (panels A-C), and $37^{\circ} \mathrm{C}(310 \mathrm{~K})$ for DMPC (D-F). Dashed lines in panels A and D are shown to guide the eye.

The plateau region of the POPC- $\mathrm{d}_{31}$ order parameters assigned to segments 2 to 5 occurs at 0.20 . The order parameter profile hardly changes upon addition of LAH4 at $\mathrm{pH} 7.5$ (Fig. 5B,C) where the peptide adopts transmembrane alignments (Figs. 1E and 3). In contrast at pH 5.1 when the peptide is in-plane (Figs. 1F and 3) a 10-15\% decrease in the order parameters is observed (Fig. 5B,C). These observations are in agreement with previous investigations of a related peptide LAH4-L1 in POPE/POPG 3/1 membranes [61].

The order parameter plateau of DMPC occurs at 0.20 (Fig. 5E). While at $\mathrm{pH} 5$, where LAH4 adopts a transmembrane orientation (Figs. 1A,B and 3), the plateau region remains unaffected by the peptide a $5 \%$ increase in the order parameter is observed upon addition of 2 mole\% LAH4 (Fig. 5F). At pH 3.1 a decrease by $3 \%$ in the order of the plateau region and an increase by about the same amount at positions 5-13 is observed (Fig. 5F). Because it has been shown that full hydration favors the transmembrane alignment of the antimicrobial 
peptide PGLa in dimyristoyl phospholipids [8] it cannot be excluded that the transition also shifts for LAH4 thus under the conditions of the ${ }^{2} \mathrm{H}$ solid-state NMR experiments the peptide potentially adopts transmembrane alignments already at $\mathrm{pH} 3.1$. Because it is difficult to maintain chemically stable samples at even more extreme $\mathrm{pH}$ values especially at full hydration this has not been investigated further.

\section{Discussion}

Lipids are important determinants for peptide topology: The prediction of the membrane topology of proteins focuses on the amino acid composition of the sequences [28, 62, 63] including previous investigations of helical model sequences using solid-state NMR [34, 36, 41]. This approach was refined by measuring the energies of transferring amino acid residues from the membrane interface to the hydrophobic interior [28, 45].

When the topology of PGLa and its synergistic interactions with magainin 2 were investigated a pronounced influence of the fatty acyl chain composition was observed where the transition of PGLa from in-planar to transmembrane only occurs in fully saturated membranes $[8,27]$. Here the influence of fatty acyl chain and phospholipid head group composition was investigated in quantitative detail for LAH4, a designer peptide resembling PGLa in its hydrophobic angle when analyzed by helical wheel representations (Figure S1). Indeed, also in the case of LAH4 the lipid head group and fatty acyl chain composition have a major effect on the $\mathrm{pH}$-dependence of its in-planar or transmembrane alignments (Figure 3).

Interaction contributions that influence IP-TM equilibria: A thermodynamic framework has previously been established to describe such transitions of helices from inplanar to transmembrane orientations. Importantly, even if exchange between the two configurations is slow on the $10^{-4}$ second time scale thus both topologies are observed simultaneously in ${ }^{15} \mathrm{~N}$ solid-state NMR spectra (e.g. spectra shown in Figures 1 and 4) previous experiments using ATR FTIR have shown that the transition is reversible [12].

The in-plane - to - transmembrane transition of a helical polypeptide domain comes along with changes in the Gibbs free energy $\Delta \mathrm{G}$ that arise from hydrophobic interactions $\left(\Delta \mathrm{G}_{\mathrm{h}}\right)$, from placing polar side-chains into the hydrophobic interior $\left(\Delta \mathrm{G}_{\mathrm{p}}\right), \mathrm{a} \mathrm{pH}$-dependent energy of discharge of the four histidine side chains $\left(\Delta \mathrm{G}_{\mathrm{d}}\right)$, conformational changes $\left(\Delta \mathrm{G}_{\mathrm{c}}\right)$ and hydrophobic mismatch $\left(\Delta \mathrm{G}_{\mathrm{m}}\right)$ ([41] and references cited therein). For example, the helical outlines of LAH4 in DPC micelles have been shown to change in a $\mathrm{pH}$ dependent manner 
[30] while the random coil to helical transition of magainin has been associated with a -0.5 $\mathrm{kJ} /$ mole per residue contribution to membrane partitioning [64].

Furthermore, it has been demonstrated that LAH4 peptides interact with each other and arrange in a mesophase arrangement when oriented along the surface of phosphatidylcholine membranes [33]. Therefore, interactions between the peptides also change during the transition and have to be taken into consideration $\left(\Delta \mathrm{G}_{\mathrm{i}}\right)$. In addition, interactions between the peptides and the lipids have an impact $\left(\Delta \mathrm{G}_{\mathrm{j}}\right)$. Another layer of complexity arises from lipophobic (entropic) $[41,65]$ and van der Waals interactions $\left(\Delta \mathrm{G}_{1}+\right.$ $\Delta \mathrm{Gw}$ ) which are related to the lipid curvature strain, the lipid structure and peptide-lipid interactions ([41] and references cited therein). Therefore, the total Gibbs free energy is influenced by a set of contributions well beyond the changes in environment of individual amino acid residues within the helical structure:

$$
\Delta \mathrm{G}=\Delta \mathrm{G}_{\mathrm{h}}+\Delta \mathrm{G}_{\mathrm{p}}+\Delta \mathrm{G}_{\mathrm{d}}+\Delta \mathrm{G}_{\mathrm{m}}+\Delta \mathrm{G}_{\mathrm{l}}+\Delta \mathrm{G}_{\mathrm{W}}+\Delta \mathrm{G}_{\mathrm{c}}+\Delta \mathrm{G}_{\mathrm{i}}+\Delta \mathrm{G}_{\mathrm{j}} \text { (equation 2) [37, 41]. }
$$

LAH4 is carrying four histidines that change their protonation state during the $\mathrm{pH}$ titration and the experimentally accessible proportion of transmembrane peptide $\mathrm{P}_{\mathrm{TM}}$ (Figure 3) has been calculated as [45]:

$$
\left.P_{T M}=\frac{[T M]}{[T M]+\left[I P^{o}\right]+\left[I P^{c h}\right]}=\frac{1}{1+e^{\frac{\Delta G}{R T}}\left(1+e^{2.3\left(p K_{a}-p H\right)}\right)^{4}} \text { (equation } 1\right)
$$

where the total peptide concentration encompasses transmembrane [TM] and in-planar states with the histdines all neutral $\left[\mathrm{IP}^{\circ}\right]$ or some of them charged [IP $\left.{ }^{\mathrm{ch}}\right]$. The $\mathrm{pK}_{\mathrm{a}}$ describes the acidbase equilibrium of the histidines. Notably, the slope of the transition is a function of $\Delta \mathrm{G}$ where close to zero or positive values result in an only partially populated transmembrane state even when the $\mathrm{pH}$ is much increased [45]. Furthermore, this theoretical framework shows that the transition midpoint is shifted to higher values due to the degeneracy of the inplanar state. The observations made with LAH4 in POPG are thus in line with this previously simulated behavior (Figure 3).

For situations where $\Delta \mathrm{G}$ is strongly negative, such as very hydrophobic peptide sequences, the transition occurs at $\mathrm{pH}$ values below the $\mathrm{pK}_{\mathrm{a}}$ namely at $p K_{a}+\frac{\Delta G}{4 \cdot 2.3 R T}$ (equation 3 ) and the sigmoidal curve can be approximated by [41, 45]

$P_{T M} \approx \frac{1}{1+e^{4 \cdot 2.3\left(p K_{a}+\frac{\Delta G}{2.3 \cdot 4 R T}-p H\right)}}$ (equation 4).

Thus, according to equation (1) the midpoint of transition, $\mathrm{pH}_{50}$, where $50 \%$ of the molecules are in the transmembrane state $\left(\mathrm{P}_{\mathrm{TM}}=1 / 2\right)$ is: $p H_{50}=p K_{a}-\frac{1}{2.3} \ln \left(e^{-\frac{\Delta G}{4 R T}}-1\right)$ (equation 5). 
From these considerations, for very negative $\Delta \mathrm{G}$ a linear correlation between the transition $\mathrm{pH}$ and the Gibbs free energy governing the in-plane - transmembrane equilibrium is obtained [45]: $\Delta p H=-\frac{\Delta \Delta G}{4 \cdot 2.3 \cdot R T}$ (equation 6).

Thereby the transition-pHs observed in bilayers of different lipid composition can be used to obtain information on the relative lipid contributions to $\Delta \mathrm{G}$. For example, in the fully saturated DMPC lipid the transition occurs at $\mathrm{pH} 3.5$ whereas it happens at $\mathrm{pH} 6.1$ in POPC where one of the fatty acyl chains is unsaturated. This $\Delta \mathrm{pH}$ translates into a difference in the driving forces for peptide realignment of these two bilayers $\Delta \Delta \mathrm{G}$ of about $60 \mathrm{~kJ} / \mathrm{mole}$. This is an energy comparable with exchanging several hydrophobic amino acids with residues that prefer an interfacial or aqueous localization [28, 34, 45]. For example, the transfer of 4-5 lysines from water to octanol has been associated with a penalty of about this size [28].

The IP to TM transition in POPG: In contrast, the in-planar topology is stabilized in POPG when compared to the PC membranes with a pK50 around 7.1 (Figure 3). Notably, in POPG the transition to transmembrane is incomplete even at $\mathrm{pH} 11$ indicating that the $\Delta \mathrm{G}$ of the transition is close to zero once the histidines have lost their charges. This has been analyzed in quantitative detail [45] and the corresponding $\mathrm{pH}$-dependent transition curves have been simulated in Figure 3. The in-plane topology is probably stabilized by the negative surface charge density and the additional hydrogen bonding possibilities of the PG bilayers that favorably interact with the histidines (and lysines). Indeed, molecular dynamics calculations show an accumulation of close contacts between cationic amino acids of membrane-inserted antimicrobial peptides and the PG phosphate groups [66, 67]. Furthermore, due to the negative surface charge density of the PG bilayer there may indeed be a pH gradient even in the reduced volume of the stacked membranes (Figure S2).

LAH4 and PGLa respond to lipid saturation: Notably, not only LAH4 but also the antimicrobial peptide PGLa, which exhibits related hydrophobic angles in helical wheel representations (Figure S1), exhibits a range of different tilt angles when studied in the presence of saturated lipids of very different hydrophobic thickness but not when one chain of the phospholipid is unsaturated $[8,38]$. The topological transition of PGLa is most pronounced in the presence of equimolar amounts of magainin where PGLa adopts tilt angles that agree with transmembrane configurations $[8,27]$. This observation could be reproduced with bilayers of different head group composition such as PE and PC membranes arguing against curvature being the major determinant for the PGLa membrane insertion [37]. In contrast magainin 2, which adopts a smaller hydrophobic angle (Figure S1) and a less 
pronounced membrane insertion $[68,69]$, adopts more stable in-plane configurations $[8,27$, 70].

Order parameters correlate with helix alignment: For LAH4, magainin 2 and PGLa a profound decrease in the ${ }^{2} \mathrm{H}$ order parameters is observed when the peptides are aligned parallel to the membrane surface, an effect which disappears for transmembrane alignments (Figure 5A-C) [37]. This observation suggests that the lipid contributions $\Delta \mathrm{G}_{1}+\Delta \mathrm{G}_{\mathrm{w}}$ play an important role for the helix orientation. An amphipathic helical peptide residing at the membrane interface takes more space at the lipid head group region when compared to the hydrophobic interior [71, 72]. Thereby, the membrane monolayer exhibits positive curvature strain, an increased disorder of the lipid fatty acyl chains (Fig. 5A-C) concomitant with membrane thinning [73]. This strain is released when the peptide adopts a transmembrane alignment concomitant with ${ }^{2} \mathrm{H}$ order parameters close to the ones in the pure lipids (Fig. 5C). Indeed, the disordering of the lipid fatty acyl chains in the presence of amphipathic helices oriented along the membrane surface has been recognized as a driving force for TM realignments [71]. Thus the LAH4 helix orientation determined by ${ }^{15} \mathrm{~N}$ solid-state NMR spectroscopy correlates well with the disordering of the palmitoyl chain of POPC and the myristoyl chains of DMPC at pH 5 (Figs. 3 and 5C,F). A good correlation between fatty acyl chain order parameters and helix alignment has also been observed for the antimicrobial peptide PGLa when investigated in a variety of membranes [37]. Because the LAH4 alignment in fully hydrated DMPC bilayers at $\mathrm{pH} 3$ remains uncertain (cf. above) this data set cannot be taken into consideration in the present discussion and the ultimate test of consistency with above hypothesis not be obtained.

Notably, for PGLa the transmembrane insertion in the presence of magainin 2 occurs in saturated PC membranes of very different thickness, in DMPC or in DMPE bilayers. This indicates that the hydrophobic thickness and the shape of the lipids are only of secondary importance in driving the helix realignment $[8,27,37]$. Therefore, while DMPC and POPC also differ slightly in thickness and overall shape the degree of saturation seems a more important parameter to explain the observed differences in the LAH4 topological transition (Fig. 3).

Quantitative considerations POPC versus DMPC: Here (Fig. 5) and in previous investigations [37] the palmitoyl chain of palmitoyl-oleoyl phospholipids and of the two fatty acyl chains in di-myristoyl phospholipids exhibit a similar degree of disorder in the presence of in-plane oriented amphipathic helices. This is somewhat surprising because PGLa and LAH4 exhibit a quite different behavior in DMPC and POPC lipids (Figure 3) [8, 27, 37]. 
Therefore, more data are needed to fully understand why when compared to POPC the fully saturated membranes favor transmembrane alignments to such an extent. Notably, there are two saturated chains in DMPC but only one in POPC. The saturated chains may act in a highly cooperative manner when aligned side-by-side in a fully saturated lipid bilayer. In contrast, in POPC the cis double bond of the unsaturated oleoyl chain probably 'decouples' the palmitoyl chains from each other by introducing considerably disorder already in the case of pure lipid bilayers. Furthermore, the per se more disordered unsaturated oleoyl chain may help to compensate for the disruption of the lipid packing upon peptide insertion.

\section{Conclusions}

The pH-dependent realignment of LAH4 occurs at a much lower $\mathrm{pH}$ in DMPC than in POPC membranes and at $\mathrm{pH}$ values well below the histidine $\mathrm{pK}_{\mathrm{a}}$. Thus, the data indicate that favorable driving forces for the in-plane - transmembrane transition arise from the saturated lipids where the in-plane alignment of amphipathic peptides causes significant membrane disorder. Theoretical analysis has shown that such disordering is a strong driving force for topological changes, oligomerization and pore formation [71, 72]. Indeed, a strong disordering of the deuterated myristoyl and palmitoyl chains by magainin 2 and PGLa has been demonstrated in a previous publication where the changes due to the membraneassociated magainin is driving PGLa realignment [37]. Here we used the LAH4 model antimicrobial peptide to monitor the $\mathrm{pH}_{50}$ of the transition which allowed us to quantitatively evaluate the Gibbs free energy difference for the peptide realignment when DMPC and POPC membranes are compared to each other. We suggest that similar contributions from lipid saturation arise also for the magainin-driven topological change of PGLa where related observations have been made but could not be evaluated in a quantitative manner [37]. Notably, the topological rearrangements that are modulated by lipid composition, another peptide or a protein domain can have an important role in the regulation of membrane polypeptides.

Author contributions: ES performed experiments, prepared Figures and helped in the writing sections of the manuscript. CA performed experiments and helped in the discussion. BB organized funding, designed the experiments, helped in the analysis and discussion and wrote the paper. 


\section{Acknowledgements}

The financial contributions of the Agence Nationale de la Recherche (projects MemPepSyn 14-CE34-0001-01, InMembrane 15-CE11-0017-01, Biosupramol 17-CE18-0033-3,

Naturalarsenal 19-AMRB-0004-02 and the LabEx Chemistry of Complex Systems 10-LABX0026_CSC), the University of Strasbourg, the CNRS, the Région Grand-Est and the RTRA International Center of Frontier Research in Chemistry are gratefully acknowledged.

\section{References}

[1] J.L. Popot, D.M. Engelman, Membrane protein folding and oligomerization: the two-stage model, Biochemistry, 29 (1990) 4031-4037.

[2] T. Pipatpolkai, D. Quetschlich, P.J. Stansfel, From Bench to Biomolecular simulation: Phospholipid Modulation of Potassium Channels, J Mol Biol, (2021) 167105.

[3] D. Hilger, The role of structural dynamics in GPCR-mediated signaling, Febs j, 288 (2021) 2461-2489.

[4] J. Lakshmaiah Narayana, B. Mishra, T. Lushnikova, Q. Wu, Y.S. Chhonker, Y. Zhang, D. Zarena, E.S. Salnikov, X. Dang, F. Wang, C. Murphy, K.W. Foster, S. Gorantla, B. Bechinger, D.J. Murry, G. Wang, Two distinct amphipathic peptide antibiotics with systemic efficacy, Proc Natl Acad Sci U S A, 117 (2020) 19446-19454.

[5] B. Bechinger, D.W. Juhl, E. Glattard, C. Aisenbrey, Revealing the mechanisms of synergistic action of two magainin antimicrobial peptides Frontiers in Medical Technology, section Pharmaceutical Innovation 2 (2020) 615494.

[6] C. Aisenbrey, A. Marquette, B. Bechinger, The Mechanisms of Action of Cationic Antimicrobial Peptides Refined by Novel Concepts from Biophysical Investigations, Adv Exp Med Biol 1117 (2019) 33-64.

[7] R.W. Glaser, C. Sachse, U.H. Durr, P. Wadhwani, S. Afonin, E. Strandberg, A.S. Ulrich, Concentration-dependent realignment of the antimicrobial peptide PGLa in lipid membranes observed by solid-state 19F-NMR, Biophys J, 88 (2005) 3392-3397.

[8] E. Salnikov, B. Bechinger, Lipid-controlled peptide topology and interactions in bilayers: structural insights into the synergistic enhancement of the antimicrobial activities of PGLa and magainin 2. , Biophysical J. , 100 (2011) 1473-1480.

[9] M. Hong, Oligomeric structure, dynamics, and orientation of membrane proteins from solid-state NMR, Structure, 14 (2006) 1731-1740.

[10] B. Bechinger, Membrane association and pore formation by alpha-helical peptides, Adv Exp Med Biol, 677 (2010) 24-30.

[11] T. Hessa, N.M. Meindl-Beinker, A. Bernsel, H. Kim, Y. Sato, M. Lerch-Bader, I. Nilsson, S.H. White, G. von Heijne, Molecular code for transmembrane-helix recognition by the Sec61 translocon, Nature, 450 (2007) 1026-1030.

[12] B. Bechinger, J.M. Ruysschaert, E. Goormaghtigh, Membrane Helix Orientation from Linear Dichroism of Infrared Attenuated Total Reflection Spectra, Biophys.J., 76 (1999) 552-563.

[13] M. Michalek, E. Salnikov, S. Werten, B. Bechinger, Structure and topology of the huntingtin 1-17 membrane anchor by a combined solution and solid-state NMR approach, Biophys. J., 105 (2013) 699-710.

[14] B. Claro, E. Goormaghtigh, M. Bastos, Attenuated total reflection-Fourier transform infrared spectroscopy: a tool to characterize antimicrobial cyclic peptide-membrane interactions, Eur Biophys J, 50 (2021) 629-639. 
[15] E.S. Salnikov, J. Raya, M. De Zotti, E. Zaitseva, C. Peggion, G. Ballano, C. Toniolo, J. Raap, B. Bechinger, Alamethicin supramolecular organization in lipid membranes from 19F solid- state NMR, Biophys J, 111 (2016) 2450-2459.

[16] W.B. Luo, R. Mani, M. Hong, Side-chain conformation of the M2 transmembrane peptide proton channel of influenza a virus from (19)F solid-state NMR, Journal of Physical Chemistry B, 111 (2007) 10825-10832.

[17] C. Aisenbrey, M. Amaro, P. Pospisil, M. Hof, B. Bechinger, Highly synergistic antimicrobial activity of magainin 2 and PGLa peptides is rooted in the formation of supramolecular complexes with lipids, Sci Rep, 10 (2020) 11652.

[18] A. Walrant, A. Bauzá, C. Girardet, I.D. Alves, S. Lecomte, F. Illien, S. Cardon, N. Chaianantakul, M. Pallerla, F. Burlina, A. Frontera, S. Sagan, Ionpair- $\pi$ interactions favor cell penetration of arginine/tryptophan-rich cell-penetrating peptides, Biochim Biophys Acta Biomembr, 1862 (2020) 183098.

[19] C. Aisenbrey, O. Rifi, B. Bechinger, Structure, membrane topology and influence of cholesterol of the membrane proximal region - transmembrane helical anchor sequence of gp41 from HIV Sci Rep, 10 (2020) 22278.

[20] C. Aisenbrey, E.S. Salnikov, B. Bechinger, Solid-State NMR Investigations of the MHC II Transmembrane Domains: Topological Equilibria and Lipid Interactions, J Membr Biol, 252 (2019) 371-384.

[21] N.A. Lakomek, J.D. Kaufman, S.J. Stahl, J.M. Louis, A. Grishaev, P.T. Wingfield, A. Bax, Internal dynamics of the homotrimeric HIV-1 viral coat protein gp41 on multiple time scales, Angew Chem Int Ed Engl, 52 (2013) 3911-3915.

[22] E.S. Salnikov, C. Aisenbrey, B. Pokrandt, B. Brugger, B. Bechinger, Structure, Topology, and Dynamics of Membrane-Inserted Polypeptides and Lipids by Solid-State NMR Spectroscopy: Investigations of the Transmembrane Domains of the DQ Beta-1 Subunit of the MHC II Receptor and of the COP I Protein p24, Front Mol Biosci, 6 (2019) 83.

[23] K. Bertelsen, B. Paaske, L. Thogersen, E. Tajkhorshid, B. Schiott, T. Skrydstrup, N.C. Nielsen, T. Vosegaard, Residue-specific information about the dynamics of antimicrobial peptides from (1)H-(15)N and (2)H solid-state NMR spectroscopy, J Am Chem Soc, 131 (2009) 18335-18342.

[24] R. Fu, Y. Miao, H. Qin, T.A. Cross, Observation of the Imidazole-Imidazolium Hydrogen Bonds Responsible for Selective Proton Conductance in the Influenza A M2 Channel, J Am Chem Soc, 142 (2020) 2115-2119.

[25] M. Mihailescu, M. Sorci, J. Seckute, V.I. Silin, J. Hammer, B.S. Perrin, Jr., J.I. Hernandez, N. Smajic, A. Shrestha, K.A. Bogardus, A.I. Greenwood, R. Fu, J. Blazyk, R.W. Pastor, L.K. Nicholson, G. Belfort, M.L. Cotten, Structure and Function in Antimicrobial Piscidins: Histidine Position, Directionality of Membrane Insertion, and pH-Dependent Permeabilization, J Am Chem Soc, 141 (2019) 9837-9853.

[26] E. Salnikov, C. Aisenbrey, V. Vidovic, B. Bechinger, Solid-state NMR approaches to measure topological equilibria and dynamics of membrane polypeptides, Biochim. Biophys. Acta 1798 (2010) 258-265.

[27] E. Strandberg, J. Zerweck, P. Wadhwani, A.S. Ulrich, Synergistic Insertion of Antimicrobial Magainin-Family Peptides in Membranes Depends on the Lipid Spontaneous Curvature, Biophysical Journal, 104 (2013) L09-L11.

[28] S.H. White, W.C. Wimley, Membrane protein folding and stability: Physical principles, Annu.Rev.Biophys.Biomol.Struct., 28 (1999) 319-365.

[29] B. Bechinger, The structure, dynamics and orientation of antimicrobial peptides in membranes by multidimensional solid-state NMR spectroscopy, Biochim. Biophys. Acta, 1462 (1999) 157-183. 
[30] J. Georgescu, V.H.O. Munhoz, B. Bechinger, NMR structures of the histidine-rich peptide LAH4 in micellar environments: membrane insertion, $\mathrm{pH}$-dependent mode of antimicrobial action and DNA transfection, Biophys J, 99 (2010) 2507-2515

[31] R. Mani, S.D. Cady, M. Tang, A.J. Waring, R.I. Lehrer, M. Hong, Membrane-dependent oligomeric structure and pore formation of a beta-hairpin antimicrobial peptide in lipid bilayers from solid-state NMR, Proc. Natl. Acad. Sci. U S A, 103 (2006) 16242-16247.

[32] E. Salnikov, C. Aisenbrey, S.V. Balandin, M.N. Zhmak, A.Y. Ovchinnikova, B. Bechinger, Structure and alignment of the membrane-associated antimicrobial peptide arenicin by oriented solid-state NMR spectroscopy, Biochemistry, 50 (2011) 3784-3795.

[33] C. Aisenbrey, B. Bechinger, Molecular Packing of Amphipathic Peptides on the Surface of Lipid Membranes, Langmuir, 30 (2014) 10374-10383.

[34] T.C.B. Vogt, P. Ducarme, S. Schinzel, R. Brasseur, B. Bechinger, The topology of lysine-containing amphipathic peptides in bilayers by CD, solid-state NMR and molecular modelling, Biophys.J., 79 (2000) 2644-2656.

[35] U. Harzer, B. Bechinger, The alignment of lysine-anchored membrane peptides under conditions of hydrophobic mismatch: A CD, $15 \mathrm{~N}$ and $31 \mathrm{P}$ solid-state NMR spectroscopy investigation, Biochemistry, 39 (2000) 13106-13114.

[36] B. Bechinger, Understanding peptide interactions with lipid bilayers: a guide to membrane protein engeneering, Curr.Opin.Chem.Biol., 4 (2000) 639-644.

[37] N. Harmouche, B. Bechinger, Lipid-mediated interactions between the amphipathic antimicrobial peptides magainin 2 and PGLa in phospholipid bilayers, Biophysical Journal, 115 (2018) 1033-1044.

[38] P. Tremouilhac, E. Strandberg, P. Wadhwani, A.S. Ulrich, Conditions affecting the realignment of the antimicrobial peptide PGLa in membranes as monitored by solid state 2H-NMR, Biochim Biophys Acta, 1758 (2006) 1330-1342.

[39] E. Strandberg, D. Horn, S. Reisser, J. Zerweck, P. Wadhwani, A.S. Ulrich, 2H-NMR and MD Simulations Reveal Membrane-Bound Conformation of Magainin 2 and Its Synergy with PGLa, Biophys J, 111 (2016) 2149-2161.

[40] K. Matsuzaki, A. Nakamura, O. Murase, K. Sugishita, N. Fujii, K. Miyajima, Modulation of magainin 2-lipid bilayer interactions by peptide charge, Biochemistry, 36 (1997) 21042111.

[41] B. Bechinger, Towards membrane protein design: $\mathrm{pH}$-sensitive topology of histidinecontaining polypeptides, J.Mol.Biol., 263 (1996) 768-775.

[42] G. Moulay, C. Leborgne, A.J. Mason, C. Aisenbrey, A. Kichler, B. Bechinger, Histidinerich designer peptides of the LAH4 family promote cell delivery of a multitude of cargo, J Pept Sci, 23 (2017) 320-328.

[43] S. Majdoul, A.K. Seye, A. Kichler, N. Holic, A. Galy, B. Bechinger, D. Fenard, Molecular Determinants of Vectofusin-1 and Its Derivatives for the Enhancement of Lentivirally Mediated Gene Transfer into Hematopoietic Stem/Progenitor Cells, J Biol Chem, 291 (2016) 2161-2169.

[44] C. Aisenbrey, R. Kinder, E. Goormaghtigh, J.M. Ruysschaert, B. Bechinger, Interactions involved in the realignment of membrane-associated helices: An investigation using oriented solid-state NMR and ATR-FTIR spectroscopies topologies J. Biol. Chem., 281 (2006) 7708-7716.

[45] C. Aisenbrey, E. Goormaghtigh, J.M. Ruysschaert, B. Bechinger, Translocation of amino acyl residues from the membrane interface to the hydrophobic core: Thermodynamic model and experimental analysis using ATR-FTIR spectroscopy, Molecular Membrane Biology, 23 (2006) 363-374.

[46] E.S. Salnikov, E. Glattard, M. Lointier, J. Raya, D.W. Juhl, A. Saad, B. Bechinger, New concepts for the mechanisms of action of antimicrobial peptides from solid-state NMR investigations. , in: A. Bhunia, H.S. Atreya, N. Sinha (Eds.) NMR Spectroscopy for 
Probing Functional Dynamics at Biological Interfaces, Royal Society of Chemistry, London, 2021 in press.

[47] B. Bechinger, C. Sizun, Alignment and structural analysis of membrane polypeptides by $15 \mathrm{~N}$ and 31P solid-state NMR spectroscopy Concepts in Magnetic Resonance, 18A (2003) $130-145$

[48] B. Bechinger, E.S. Salnikov, The membrane interactions of antimicrobial peptides revealed by solid-state NMR spectroscopy, Chem Phys Lipids, 165 (2012) 282-301.

[49] C. Aisenbrey, P. Kemayo-Koumkoua, E.S. Salnikov, E. Glattard, B. Bechinger, Investigations of the structure, topology and interactions of the transmembrane domain of the lipid sorting protein $\mathrm{p} 24$ being highly selective for sphingomyelin-C18 Biochemistry, 58 (2019) 2782-2795.

[50] C. Aisenbrey, M. Michalek, E.S. Salnikov, B. Bechinger, Solid-state NMR approaches to study protein structure and protein-lipid interactions, in: J.H. Kleinschmidt (Ed.) LipidProtein Interactions: Methods and Protocols, Springer, New York, 2013, pp. 357-387.

[51] M. Rance, R.A. Byrd, Obtaining High-Fidelity Spin-1/2 Powder Spectra in Anisotropic Media: Phase-Cycled Hahn Echo Spectroscopy, Journal of Magnetic Resonance, 52 (1983) 221-240.

[52] J.H. Davis, K.R. Jeffrey, M. Bloom, M.I. Valic, T.P. Higgs, Quadrupolar Echo Deuteron Magnetic Resonance Spectroscopy in Ordered Hydrocarbon Chains, Chem.Phys.Lett., 42 (1976) 390-394.

[53] P.L. Gor'kov, E.Y. Chekmenev, C. Li, M. Cotten, J.J. Buffy, N.J. Traaseth, G. Veglia, W.W. Brey, Using low-E resonators to reduce RF heating in biological samples for static solid-state NMR up to 900 MHz, J Magn Reson, 185 (2007) 77-93.

[54] B. Bechinger, S.J. Opella, Flat-Coil Probe for NMR Spectroscopy of Oriented Membrane Samples, J.Magn.Reson., 95 (1991) 585-588.

[55] B.M. Fung, A.K. Khitrin, K. Ermolaev, An improved broadband decoupling sequence for liquid crystals and solids, J. Magn. Reson., 142 (2000) 97-101.

[56] P. Bertani, J. Raya, B. Bechinger, 15N chemical shift referencing in solid state NMR, Solid-state NMR spec. , 61-62 (2014) 15-18

[57] M. Bloom, Davis, J.H., Mackay, A.L., Direct determination of the oriented sample NMR spectrum from the powder spectrum for the systems with local axial symmetry Chem. Phys. Letters, 80 (1981) 198-202.

[58] L.S. Batchelder, H. Niu, D.A. Torchia, Methyl reorientation in polycrystalline amino acids and peptides: A 2H NMR spin lattice relaxation study, J.Am.Chem.Soc., 105 (1983) 2228-2231.

[59] R. Storm, Wahrscheinlichkeitsrechnung, Mathematische Statistik, Statistische Qualitätskontrolle, VEB Fachbuchverlag, Leipzig, 1967.

[60] B. Perrone, A.J. Miles, E.S. Salnikov, B. Wallace, B. Bechinger, Lipid- interactions of the LAH4, a peptide with antimicrobial and nucleic transfection activities Eur. Biophys. J. , 43 (2014) 499-507.

[61] E.S. Salnikov, A.J. Mason, B. Bechinger, Membrane order perturbation in the presence of antimicrobial peptides by $2 \mathrm{H}$ solid-state NMR spectroscopy, Biochimie, 91 (2009) 734-743.

[62] J. Kyte, R.F. Doolittle, A Simple Method for Displying the Hydropathic Character of a Protein, J.Mol.Biol., 157 (1982) 105-132.

[63] T. Hessa, H. Kim, K. Bihlmaier, C. Lundin, J. Boekel, H. Andersson, I. Nilsson, S.H. White, G. von Heijne, Recognition of transmembrane helices by the endoplasmic reticulum translocon, Nature, 433 (2005) 377-381.

[64] T. Wieprecht, O. Apostolov, M. Beyermann, J. Seelig, Thermodynamics of the alphahelix-coil transition of amphipathic peptides in a membrane environment: implications for the peptide-membrane binding equilibrium, J Mol Biol, 294 (1999) 785-794. 
[65] J.P. Duneau, J.N. Sturgis, Lateral organization of biological membranes: role of longrange interactions, Eur Biophys J, 42 (2013) 843-850.

[66] F. Ramos-Martin, N. D'Amelio, Molecular Basis of the Anticancer and Antibacterial Properties of CecropinXJ Peptide: An In Silico Study, Int J Mol Sci, 22 (2021).

[67] F. Ramos-Martin, C. Herrera-Leon, V. Antonietti, P. Sonnet, C. Sarazin, N. D'Amelio, Antimicrobial Peptide K11 Selectively Recognizes Bacterial Biomimetic Membranes and Acts by Twisting Their Bilayers, Pharmaceuticals (Basel), 14 (2020).

[68] E. Glattard, E.S. Salnikov, C. Aisenbrey, B. Bechinger, Investigations of the synergistic enhancement of antimicrobial activity in mixtures of magainin 2 and PGLa, Biophys Chem, 210 (2016) 35-44.

[69] M. Pachler, I. Kabelka, M.S. Appavou, K. Lohner, R. Vacha, G. Pabst, Magainin 2 and PGLa in Bacterial Membrane Mimics I: Peptide-Peptide and Lipid-Peptide Interactions, Biophys J, 117 (2019) 1858-1869.

[70] B. Bechinger, Insights into the mechanisms of action of host defence peptides from biophysical and structural investigations, J Pept Sci, 17 (2011) 306-314.

[71] A. Zemel, A. Ben-Shaul, S. May, Perturbation of a lipid membrane by amphipathic peptides and its role in pore formation, Eur Biophys J, 34 (2005) 230-242.

[72] H. Aranda-Espinoza, A. Berman, N. Dan, P. Pincus, S. Safran, Interaction between inclusions embedded in membranes, Biophys J, 71 (1996) 648-656.

[73] S. Ludtke, K. He, H. Huang, Membrane thinning caused by magainin 2, Biochemistry, 34 (1995) 16764-16769. 